\title{
CONCLUSIONS AND POLICY RECOMMENDATIONS FOR THE E.U. WATER FRAMEWORK DIRECTIVE
}

\author{
Katia Karousakis, Phoebe Koundouri, Dionysis Assimacopoulos, Paul Jeffrey, and \\ Manfred Lange
}

This book has presented a selection of results from the ARID Cluster of projects. The three projects have explored aspects of water scarcity and demand in arid and semi-arid regions, as well as participatory and adaptive approaches to defining and applying appropriate management strategies. Given the importance, multiple uses, and competing demands for water, we have adopted an interdisciplinary perspective on water resources management. More specifically, the adopted perspectives have included an engineering approach, namely the use of a Decision Support System that can simulate water supply and demand under different scenarios and management options and strategies (Part I); a stakeholder participatory approach for addressing conflicting demands and varying hydrological conditions for the sustainable use of water on Mediterranean Islands (Part II); an environmental economic perspective on efficient integrated water resources management (Part III); and finally, a co-evolutionary approach to adaptive, integrated management under changing utilisation condition (Part IV).

Part I of the foregoing text emphasised the need for a systematic evaluation of water management interventions over extended timescales, simulating long-run accumulative effects and anticipating potential future changes and uncertainties. Following an analysis of water management practices and problems in different regions, and the nalysis of stakeholder perspectives for addressing water planning, the adopted methodology employs a GIS based Decision Support System that enables simulation of alternative water availability and demand scenarios. In contrast to previous methodologies and other DSS, the WaterStrategyMan DSS allows a more thorough incorporation of social, 
environmental, economic and technological dimensions and interrelationships in the simulation analysis. The application of the DSS platform to a case study in Portugal illustrates how the DSS can be used in other parts of the E.U. as well and how it might inform the implementation of the E.U. Water Framework Directive (WFD).

The main conclusions of Part II are that the implementation of indicators facilitates an integrated approach which is able to combine the natural and socio-economic aspects of water management while allowing stakeholder involvement. Following the Driving forces-Pressures-States-Impacts-Responses (DPSIR) approach on the islands of Corsica, Crete, Cyprus, Majorca and Sicily, the policy recommendations include demand management improvements (water saving and storage measures), institutional policy and capacity building (especially in the agricultural sector), as well as the introduction of a differentiated pricing systems amongst water users and public awareness and educational programs.

In Part III, the challenge of integrated sustainable water management is addressed from the field of environmental and resource economics. The importance of the efficient (or optimal) pricing of this resource is emphasised, and a non-technical description of the valuation methods to account for the total economic value of water is provided. The appropriate choice of economic instruments to provide users with the correct incentives for consumption will vary from region to region, depending on for example the metering technology that is available and the likely size of transaction costs associated with the different methods. The transaction costs associated with tradable water permits for example is likely to vary from one country to another depending on administrative capacity, the number of participants in the market, the existence of brokers and other issues, making such trading more or less favourable from one country to another. An important and generic conclusion for all policy-makers involved in making decisions that have long-term implications (i.e. greater than 30 years) is that they should consider the application of a declining discount rate, as opposed to the more traditional flat discount rate, to obtain net present values of potential projects. 
Part IV examines the relationships between natural resource availability and the development of human societies. The first of these chapters analysed the socio-cultural determinants of water utilisation in more detail by conducting four survey-based case studies. Findings suggest commonalities in behavioural patterns with respect to consumer perceptions regarding the availability of water, and the quality of information and education regarding water scarcity. In contrast, the surveys indicate that different cultures are likely to be more conducive to certain policy instruments over others. In the final chapter, structural change in both natural as well as social systems is analysed, as is the nature of the reciprocal co-dynamic processes that promote both ecological and social adaptive capacity in the Marina Baja region of Spain.

The purpose of this final chapter is to identify the common themes of the three projects (WSM, Medis, and Aquadapt), to integrate and harmonise the results and approaches adopted by each, and to discuss conclusions and potential policy implications that can feed into the EU Water Framework Directive. The knowledge that has emerged from the three projects has been principally obtained from case study analyses. These have examined competing water use patterns, compared governance structures and how these have evolved in response to scarcity and structural and non-structural instruments to address water deficiency. The experiences and lessons learned from these studies are summarised below. First however we briefly describe the general circumstances, or pressures and driving forces, that are common to the case studies examined in the ARID cluster of projects.

Most of the case studies examined lie in the Mediterranean region and are thus subject to similar climatic conditions. Some countries are more vulnerable to changes in water availability than others. Cyprus for example is classified as a semi-arid environment, whereas Corsica is categorised as a dry sub-humid environment. The various countries, regions, and islands studied vary with respect to characteristics such as population density, the amount of tourism they handle on a seasonal/annual basis, agricultural dependence and the types of crops that are grown. However, it is seasonal and geographical variations and differences in water availability throughout the year that 
make water planning and availability difficult, especially in the summer due to high demand for water from the tourism sector.

The importance of taking a holistic approach to the issue of sustainable water resources management is a common feature of all aspects of the projects. This is typified by the material contained in Part I of the book where the described DSS includes economic, hydrological and environmental components to evaluate the different water management options and strategies. Part II emphasises the importance of examining the physical and environmental, the economic and regulatory, and the social, institutional, and political aspects related to water management. Part III presents the methods that have been developed to evaluate environmental and social goods and services that do not have market prices in economic terms, thus enabling a more accurate reflection of costs and benefits to aid in policy-making decisions. Finally, Part IV examines how water uses are inter-related and affected by a number of different factors, namely by landscape changes, management systems and governance co-dynamics in S.E. Spain, and four case studies are undertaken in different parts of Europe to examine the socio-cultural determinants of water usage. Within this, various technological and economic approaches to water management are also addressed.

The holistic approach is important because water will need to serve a multitude of different purposes and contributes disproportionately to lives and livelihoods across a landscape. From an economic and development perspective, water affects the wider economy and society, including land-use changes and settlement growth, and changes in agricultural and industrial activity. From an environmental perspective, water provides recreational benefits and most importantly life-support functions for wildlife and of course, human health. Examining water scarcity from a holistic, multi-disciplinary perspective and involving different stakeholders ensures that a full consideration of relevant influences and options for action are considered. 
The importance of demand-side management in addition to the more traditional supply side management of water scarcity is another common underlying theme of the projects. It is becoming increasingly clear that, in the face of continued urban growth, water quality degradation, and new uncertainties brought on by climate change, supply-side mechanisms for water scarcity are unlikely to yield long-term sustainable solutions. Demand-side management options such as the efficient use of water through the reduction of losses, conservation, and water recycling and re-use will become a more important component of sustainable water management. Demand-side management will entail a change in individual attitudes, and hence behaviour, towards water use such that this is more efficient and water use more cost-effective. Policy recommendations for the improved efficiency of water use that derive from the ARID Cluster case studies include the following:

- The adoption of new technologies for reducing consumption;

- The modification of crop patterns in agriculture to reduce water use;

- The use of indicators to identify significant inefficiencies; and

- The alignment of policies in other sectors (e.g.. the elimination of subsidies to energy used for pumping groundwater for irrigation).

With regard to water conservation, re-use and recycling, the main policy recommendations that have come out of the case studies are the application of the following:

- Rain water harvesting,

- The reduction of evapo-transpiration by covering open reservoirs where this is feasible, and

- Waste water recycling and the utilisation of reclaimed and brackish water.

For the latter, these will likely need to be encouraged via the use of educational programs for farmers to accept treated sewage water, and the imposition of lower tariffs than those to surface and groundwater to provide economic incentives for its use. 
Integral to demand management is the application and use of economic instruments. Policy-makers should begin to implement water pricing programs that go beyond the objective of revenue generation to actually inducing a reduction in water wastage and promoting water-saving behaviour instead. These can be achieved via the introduction of appropriate water prices. In addition, pilot programs can be set up to obtain experience with other economic instruments such as tradable water permit systems, as has been done in the U.S., Australia, Canada, Chile, and other countries (Easter, Dinar, Rosegrant, 1998). Lessons can be learned from previous experiences and shortcomings.

Sustainable water management in the agricultural sector may deserve special attention given that in the major Mediterranean islands it uses up more than 50 percent of available water resources, and further it is the least efficient sector in water use since irrigation efficiency is in some cases less than 55 percent. The policy recommendations here are clear and straightforward. Sustainable water management in agriculture can be achieved by adopting improvements in irrigation practices, best soil and crop management practices, water pricing, the reuse of marginal waters (reclaimed or brackish) for irrigation, wider and more effective participation of farmers in water management, and capacity-building. For these policies to work effectively, governments or policy-makers will need to ensure that any existing distortionary subsidies for agricultural production are eliminated.

The need for effective monitoring and enforcement, as well as the consistent application of monetary and civil penalties when existing regulations have been breached is important for effective water management. Good administrative capacity, institutional coordination and economic stability within a country are also prerequisites for the successful application of efficient water management policies and measures.

From a social perspective, it is clear that intra- and inter-generational equity with respect to water use is also of vital importance. With regard to intra-generational equity, this applies to both within a particular sector and between sectors. For example, within 
the domestic sector, low-income and marginal households will still need to be able to afford a secure supply of water, despite the introduction of a more efficient water pricing system (which will often entail higher water prices). Similarly, between sectors, many of the case studies show a distinct conflict between the agricultural and farming sector vis-àvis the tourism sector. Equity considerations are also necessary. With regard to intergenerational equity, and the selection of projects over long-time horizons, the use of an appropriate discount rate in a cost-benefit analysis is key. The declining discount rate is arguably the most equitable manner for incorporating both current and future generations into the decision-making process.

Public awareness, educational campaigns and outreach are important as well so as to change and correct public perceptions. The four case studies that were undertaken in France, the UK, Spain and Slovenia to establish why and under what circumstances individuals are willing to alter or modify their water usage patterns indicate that those who are most concerned about water quantity and quality issues are those who claim to be the most active in water saving behaviour. Similarly, those who are most informed on water issues also tend to be those who are most active in water saving behaviour. This form of coherent behaviour is common across all of the four case studies, suggesting that public awareness and educational programs should have positive effects towards attaining sustainable water consumption and a raised awareness for sensible water use at the European level as well.

In contrast, the study reported in Chapter 11 finds that the demand management and policy prescriptions adopted in a country should be adapted to the local water cultures within each country. For example, it is concluded that local cultures across countries have different preferences with regard to management policies and prescriptions: The British for example are more willing to accept higher water bills for the protection of the environment, whereas the French are against this and would prefer to change their water consumption patterns to off-peak hours. The policy implications this has for the implementation of the EU Water Framework Directive is that some policies may vary in 
their acceptability across countries, whether this is due to existing previous experience with similar measures or other differences in the local culture. This may indicate that some policies may also be more successful in certain countries than in others, and that the decision-maker should take these considerations into account when selecting the appropriate policy responses to provide incentives for efficient water resources use.

It seems appropriate here to examine potential next steps and to conclude with some recommendations for future research. Two issues seem particularly important:

(a) Additional resources should be allocated to the collection and monitoring of accurate household, firm-level and farm-level water data; and (b) local and regional water pricing pilot schemes should be introduced in different areas to obtain experience with various economic instruments. These will enable empirical studies to be undertaken that evaluate the effectiveness of various economic instruments ${ }^{1}$ under different scenarios which can feed directly into tangible policy recommendations of the Water Framework Directive at the European level.

\footnotetext{
${ }^{1}$ Similar European studies that have focused on the effectiveness of economic instruments for air emissions include those by Sterner and Hoeglund (2000), and Millock and Nauges (2003)
} 


\section{References:}

1) Easter, W., Dinar, A., and Rosegrant, M. 1998. Markets for Water: Potential and Performance. Kluwer Academic Publishers

2) Millock, K. and Nauges. C. 2003. Ex Post Evidence on Environmental Taxes: An Assessment of the French Tax on Air Pollution. FEEM Working Paper 44.03

3) Sterner T and Hoeglund L. 2000. Output Based Refunding of Emissions Payments: Theory, Distribution of Costs, and International Experience. RFF discussion paper 00-29. Resources For the Future, Washington D.C. 